\title{
Utilisation of tracheostomy in patients with COVID-19 in England: patient characteristics, timing and outcomes
}

\author{
Annakan Navaratnam ${ }^{1}$, William Gray ${ }^{2}$, Josh Wall ${ }^{2}$, Arun Takhar ${ }^{3}$, Taran Tatla ${ }^{4}$, Jamie \\ Day $^{2}$, Anna Batchelor ${ }^{2}$, Michael Swart ${ }^{2}$, Christopher Snowden ${ }^{2}$, Andrew Marshall ${ }^{5}$, and \\ Tim Briggs ${ }^{2}$ \\ ${ }^{1}$ Royal National Throat Nose and Ear Hospital \\ ${ }^{2}$ NHS England \\ ${ }^{3}$ Guy's and St Thomas' NHS Foundation Trust \\ ${ }^{4}$ Northwick Park Hospital \\ ${ }^{5}$ Queen's Medical Centre
}

December 7, 2021

\begin{abstract}
Objectives: We aimed to characterise the use of tracheostomy procedures for all COVID-19 critical care patients in England and to understand how patient factors and timing of tracheostomy affected outcomes. Design: A retrospective observational study using exploratory analysis of hospital administrative data. Setting: All 500 National Health Service hospitals in England. Participants: All hospitalised COVID-19 patients aged [?] 18 years in England between March 1st and October 31st, 2020 were included. Main outcomes and measures: This was a retrospective exploratory analysis using the Hospital Episode Statistics administrative dataset. Multilevel modelling was used to explore the relationship between demographic factors, comorbidity and use of tracheostomy and the association between tracheostomy use, tracheostomy timing and the outcomes. Results: In total, 2,200 hospitalised COVID-19 patients had a tracheostomy. Tracheostomy utilisation varied substantially across the study period, peaking in April-June 2020. In multivariable modelling, for those admitted to critical care, tracheostomy was most common in those aged 40-79 years, in males and in people of Black and Asian ethnic groups and those with a history of cerebrovascular disease. In critical care patients, tracheostomy was associated with lower odds of mortality (OR: 0.514 (95\% CI 0.443 to 0.596 ), but greater length of stay (OR: 41.143 (95\% CI 30.979 to 54.642). In patients that survived, earlier timing of tracheostomy ([?] 14 days post admission to critical care) was significantly associated with shorter length of stay. Conclusions: Tracheostomy is safe and advantageous for critical care COVID-19 patients. Early tracheostomy may be associated with better outcomes, such as shorter length of stay, compared to late tracheostomy.
\end{abstract}

Title

Utilisation of tracheostomy in patients with COVID-19 in England: patient characteristics, timing and outcomes

\begin{abstract}
Objectives: We aimed to characterise the use of tracheostomy procedures for all COVID-19 critical care patients in England and to understand how patient factors and timing of tracheostomy affected outcomes.
\end{abstract}

Design: A retrospective observational study using exploratory analysis of hospital administrative data.

Setting: All 500 National Health Service hospitals in England. 
Participants: All hospitalised COVID-19 patients aged [?] 18 years in England between March $1^{\text {st }}$ and October $31^{\text {st }}, 2020$ were included.

Main outcomes and measures: This was a retrospective exploratory analysis using the Hospital Episode Statistics administrative dataset. Multilevel modelling was used to explore the relationship between demographic factors, comorbidity and use of tracheostomy and the association between tracheostomy use, tracheostomy timing and the outcomes.

Results: In total, 2,200 hospitalised COVID-19 patients had a tracheostomy. Tracheostomy utilisation varied across the study period, peaking in April-June 2020. In multivariable modelling, for those admitted to critical care, tracheostomy was most common in those aged 40-79 years, in males and in people of Black and Asian ethnic groups and those with a history of cerebrovascular disease. In critical care patients, tracheostomy was associated with lower odds of mortality (OR: 0.514 (95\% CI 0.443 to 0.596), but greater length of stay (OR: 41.143 (95\% CI 30.979 to 54.642). In patients that survived, earlier timing of tracheostomy ([?] 14 days post admission to critical care) was significantly associated with shorter length of stay.

Conclusions: Tracheostomy is safe and advantageous for critical care COVID-19 patients. Early tracheostomy may be associated with better outcomes, such as shorter length of stay, compared to late tracheostomy.

Key words: tracheostomy, COVID-19, SARS-CoV2, intubation, mechanical ventilation

\section{Key points}

- To our knowledge, our study is one of the largest published to date on the profile of COVID-19 tracheostomy patients.

- There was increased utilisation of tracheostomy as the COVID-19 pandemic progressed.

- Tracheostomy was beneficial procedure for COVID-19 patients requiring ventilatory weaning.

- Tracheostomy patients had lower odds of mortality and greater length of stay compared to nontracheostomy critical care patients.

- In patients that survived, earlier timing of tracheostomy was significantly associated with shorter length of stay.

INTRODUCTION Critical care admission is common in people hospitalised with COVID-19. ${ }^{1}$ In the United Kingdom, it has been reported that 17\% of COVID-19 hospital inpatients required critical care support and $10 \%$ required mechanical ventilation. ${ }^{2}$ The management of these patients has evolved as new evidence regarding treatment approaches has been developed. However, an area of controversy that has remained is the appropriate utilisation of tracheostomy and its subsequent management.

Early in the pandemic, guidelines were published based on expert opinion before much was understood about the disease. Most of these guidelines focused on minimising risk of nosocomial transmission to clinicians and delaying or avoiding tracheostomies in these patients as the benefits of the procedure were unknown. ${ }^{3,4}$ As further experience was gained, tracheostomy use became common for critical care COVID-19 patients ${ }^{5}$ although optimal timing of tracheostomy remains a subject of debate. ${ }^{6,7}$

In England, reports of departments' experiences have been described ${ }^{8-10}$ and a UK multi-centre prospective cohort study, COVIDTrach has been evaluating outcomes of COVID-19 tracheostomy patients. ${ }^{11}$ However, capturing information directly from hospitals can be constrained by inconsistent reporting patterns, especially if individual hospitals are overwhelmed by surges of critical care patients.

The National Health Service (NHS) Hospital Episodes Statistics (HES) database is an administrative dataset that contains a wide range of details regarding all NHS-funded hospital admissions in England. Using HES data, the aim of this study was to characterise tracheostomy use for COVID-19 critical care patients in England, understand the patient factors associated with having a tracheostomy and determine how this related to outcomes.

\section{METHODS}




\section{Ethics}

The analysis of data follows current NHS Digital guidance for the use of HES data for research purposes. Reported data are anonymised to the level required by ISB1523 Anonymisation Standard for Publishing Health and Social Care Data. ${ }^{12}$

\section{Study design and data collection}

This was a retrospective analysis of HES administrative data following STROBE guidelines. HES data are collected by NHS Digital for all NHS-funded patients admitted to hospitals in England.

\section{Timing, case ascertainment, inclusion and exclusion criteria}

We reviewed HES data for all completed episodes of hospital care in England with a discharge date from $1^{\text {st }}$ March to $31^{\text {st }}$ October 2020 that involved a diagnosis of COVID-19. Patients aged $<18$ years were excluded. Cases of COVID-19 were identified using the International Statistical Classification of Disease and Related Health Problems $10^{\text {th }}$ edition (ICD-10) codes U071 and U072.

Critical care (high dependency unit (HDU) or intensive care unit (ICU)) admissions and those receiving advanced respiratory support were identified.

\section{Outcomes}

Primary outcome: Tracheostomy use in critical care patients. Use of a tracheostomy was recorded if the Office of Population Censuses and Surveys Classification of Interventions and Procedures version 4 (OPCS-4) code E42- was used anywhere in the HES record of procedures.

Secondary outcomes: In-hospital mortality, length of hospital stay, length of critical care stay and tracheostomy malfunction. In these analyses, tracheostomy use was treated as the primary exposure variable. Mortality was taken from the Office for National Statistics (ONS) date linked to HES data at a patient level. An in-hospital death was recorded if the date of death was the same as or +/- one day of the date of hospital discharge recorded in HES. Tracheostomy malfunction was recorded where the ICD-10 code J950 was used.

\section{Covariates}

Age: Categorised as 18-39 years, 40-49 years, 50-59 years, 60-69 years, 70-79 years and [?] 80 years for exploratory analysis (in line with previous reports) ${ }^{13}$ and treated as continuous in the final multivariate model.

Sex: Male or female.

Ethnicity: Coded in categories used by NHS Digital: White, South Asian (Bangladeshi, Indian, Pakistani), Other Asian, Black, Mixed, Other, not stated.

Deprivation: Recorded using the Index of Multiple Deprivation (IMD) for the Lower Super Output Area (LSOA) of the patients' home address, with scores categorised into quintiles based on national averages.

Comorbidities: These were the 14 comorbidities used to construct the Charlson Comorbidity Index (Table 1).$^{14}$ The comorbidity was deemed present if it was recorded in HES as a secondary diagnosis in the index admission or as a primary or secondary diagnosis in any admission during the previous year, in accordance with the recommendations of Quan et al.$^{15}$

Obesity: Recorded as present if the ICD-10 code E66 was used as a diagnostic code during the admission.

Discharge date: Categorised into day or month of discharge (starting from $1^{\text {st }}$ March) depending on the analysis undertaken. Monthly data were used for descriptive statistics. Daily data were used for the final multivariable model.

Admission date: Categorised into month of admission. Only admissions from $1^{\text {st }}$ February to $31^{\text {st }}$ August 2020 were used, to avoid biasing the data at the end of the study period due to many patients admitted in 
September and October still being in hospital on $31^{\text {st }}$ October.

\section{Grouping variable}

NHS region: London, South-East, South-West, East of England, Midlands, North-West and North-East \& Yorkshire.

\section{Data management and statistical analyses}

Data were extracted onto a secure encrypted server controlled by NHS England and NHS Improvement. Analysis within this secure environment took place using standard statistical software: Microsoft Excel (Microsoft Corp, Redmond, WA, USA), Stata (StataCorp LLC, College Station, TX, USA) and Alteryx (Alteryx Inc, Irvine, CA, USA).

In descriptive analysis, data were categorised as detailed above and summarised in terms of frequency and percentage. Length of stay was non-normally distributed, with a right-skew, and summarised using the median and inter-quartile range (IQR).

To explore variables associated with having a tracheostomy and the association between tracheostomy and in-hospital mortality and length of stay greater than the median (8 days) in critical care patients, a series of multilevel logistic regression models were fitted using themelogit command. Two-level intercept only models were used, allowing adjustment for clustering of patients within hospital trusts. Covariates were categorised as described above. Adjusted tracheostomy and mortality rates were calculated using the margins command in Stata based on the conditional probability across the entire dataset.

For the outcomes time from tracheostomy to hospital discharge and critical care discharge a multi-level linear model was fitted based on the natural logarithm of the outcome using the mixed command in Stata. The main exposure variable of interest (timing of tracheostomy post-critical care admission) was modelled as a binary variable with a threshold of [?] 14 days with all covariates modelled as described previously.

Missing data were uncommon. No attempt was made to impute missing values. Where data were missing the numbers involved are given.

\section{RESULTS}

The data extraction process yielded a dataset of 132,446 unique patients who had a diagnosis of COVID-19 either on admission or during their stay. Of these 13,401 (10.1\%) were admitted to critical care as part of a hospital stay. Of those admitted to critical care, 7,993 (59.6\%) had advanced respiratory support and 2,200 $(16.4 \%)$ had a tracheostomy procedure recorded.

Tracheostomy use in critical care patients changed markedly over time. Use of tracheostomy and associated mortality rates for month of discharge are presented in Figure 1, tracheostomy use for each region over time by month of discharge are presented in Figure 2. By discharge date, tracheostomy rates were very low for patients discharged in March (2.6\%), peaked for discharges in June (36.7\%) and declined thereafter to $4.1 \%$ in October. This trend was seen across all regions. Mortality rates in those with a tracheostomy revealed a mirror image trend, with the lowest death rates seen at times of highest tracheostomy use. When plotted by admission month the pattern is similar, but with an earlier and smaller peak in the proportion of patients with a tracheostomy (11.9\% in February, $20.5 \%$ in April). However, there was a similar decline in the proportionate use of tracheostomy in late summer 2020 (3.8\% in August).

The profile of those admitted to hospital, those admitted to critical care and those who had advanced respiratory support and a tracheostomy is summarised in Table $\mathbf{1}$. Those admitted to critical care were more likely to be aged 40-69 years and less likely to be aged 70 years and over than the general hospitalised population. They were also more likely to be male and from a non-White ethnic background. The deprivation profile of those admitted to critical care reflected the wider population. Obese patients were over-represented in those admitted to critical care and patients with dementia, cardiovascular disease, renal disease and cancer were under-represented. The profile of those who were recorded as having a tracheostomy was similar to 
the wider critical care population, although there was a smaller percentage of people aged 70 years and over with a tracheostomy.

Factors associated with having a tracheostomy for those admitted to critical care were explored using multilevel logistic regression and the results are presented in Table 2 . Compared to the 18-39 years age group, tracheostomy was significantly more common in the 40-79 years age group and significantly less common in the 80 years and over age group. Tracheostomy was significantly more common in males, in Asian and Black ethnic groups and in patients with cerebrovascular disease. Tracheostomy was less common in patients with peripheral vascular disease, chronic heart failure, acute myocardial infarction, connective tissue/rheumatic disease, moderate/severe liver disease, renal disease and cancer.

In patients admitted to critical care, outcomes for those with and without a tracheostomy are presented in Table 3 with the adjusted association of tracheostomy with each outcome. Tracheostomy was significantly associated with reduced odds of in-hospital mortality and increased odds of length of stay greater than the median after adjusting for covariates.

The time from hospital admission to critical care admission was the same for both those who survived to discharge and those who died during their stay: median 1 day (IQR 0 to 3). Of those with a tracheostomy, $120(5.5 \%)$ had tracheostomy malfunction recorded during their stay. Of those with a malfunction recorded, 36 died in hospital (30.0\%) and in the 2080 without a malfunction 437 (21.0\%) died in hospital.

Of the 2,200 patients with a tracheostomy, data on time from critical care admission to tracheostomy were available for $1,777(80.8 \%)$ patients. Data on the timing of tracheostomy from critical care admission are presented in Table 4 for those who died and those who survived to discharge. Patients who underwent a tracheostomy at [?] 14 days from critical care admission and survived to discharge had a shorter hospital and critical care stay both overall and post-tracheostomy. Undergoing a tracheostomy [?] 14 days from critical care admission was associated with significantly shorter time from tracheostomy to critical care discharge $(\beta$ $=-0.100(95 \%$ CI -0.170 to -0.031$)$ and hospital discharge $(\beta=-0.061$ (95\% CI -0.115 to -0.007$)$.

\section{Discussion}

Our study is one of the largest published to date on the profile of COVID-19 tracheostomy patients. Approximately one in six COVID-19 critical care patients had a tracheostomy. In critical care patients, tracheostomy was associated with half the odds of mortality compared with critical care patients without tracheostomy but was associated with much longer stay.

Tracheostomy is a beneficial procedure for COVID-19 patients requiring ventilatory weaning.

In our study having a tracheostomy was associated with lower in-hospital mortality after adjustment for covariates. Patients selected for tracheostomy will have been expected to meet specific criteria in terms of lower ventilatory requirements and potential for rehabilitation. Therefore, this finding is caveated as patient selection will have influenced it. Patients selected for tracheostomy insertion will be expected to survive and consequently there will be an inherent bias in comparing outcomes of patients with and without a tracheostomy. This is demonstrated by reduced odds of COVID-19 patients with multiple co-morbidities having a tracheostomy in our cohort (Table 2). However, despite early scepticism for its role in critically ill COVID-19 patients, our study adds further evidence that tracheostomy is a beneficial intervention for COVID-19 patients requiring ventilatory weaning. ${ }^{16}$

\section{Timing of tracheostomy}

Our data demonstrated that patients that underwent early tracheostomy had shorter subsequent lengths of hospital stay. There are a variety of clinical factors including disease severity, medical therapy and emerging evidence about viral load and infectivity, which will have influenced decisions regarding timing of tracheostomy. The distinct categorisation of patients into early and late tracheostomies, even when outcomes are adjusted for patient factors, may be too simplistic. However, our data suggests that there is no need to delay tracheostomy insertion in the expectation that this will improve patient outcomes. Indeed, 
there is evidence that delaying tracheostomy insertion may lead to long-term complications such as tracheal stenosis. ${ }^{17}$

Increased utilisation of tracheostomy as the pandemic progressed.

We found that the proportion of critical care patients having a tracheostomy significantly increased during the early part of the pandemic. Anecdotally, during February-March 2020 there was some hesitation in performing tracheostomies for COVID-19 patients owing to the uncertainly regarding clinical prognosis and concerns for healthcare worker safety when performing an aerosol generating procedure. There were low rates of tracheostomies internationally during this period with early reports from the USA stating that only $8 \%(17 / 203)$ of patients had a tracheostomy in a multicentre cohort from March 2020. ${ }^{18}$

Tracheostomy use rapidly increased in April-June following the peak of critical care admissions during the first wave in England. This increase in tracheostomy utilisation reflects rapidly changing critical care practice as understanding of the disease improved and patients survived longer whilst intubated and ventilated. Furthermore, outcomes for tracheostomy patients improved as procedure numbers increased over time. It is possible that a combination of improved overall critical care management with new information and better patient selection for tracheostomy contributed to improved outcomes during early summer 2020 .

The differences when plotting the data by discharge and admission month appear to be driven by longer stay for patients undergoing tracheostomy compared to non-tracheostomy patients, with the peak in tracheostomy patient discharges in June 2020. Nevertheless, the same broad trend is evident when plotting the data by either method.

\section{Strengths and limitations}

HES data covers all NHS-funded hospital activity in England. As such they are the most complete and detailed record of hospital activity in England related to COVID-19. The eight-month study period allowed us to look at temporal trends over an extended period.

However, as with any administrative dataset, there are limitations in using HES data. HES relies on individual hospital trusts compiling data accurately and in a consistent manner. As such, some patients who underwent a tracheostomy may not have been coded as such and so number of tracheostomies reported here is likely to be an under-estimate. Furthermore, tracheostomy complications are likely to be underreported due to coding limitations. ${ }^{19}$ However, we have no reason to suspect that our data are systematically biased in terms of temporal trends or the profile of patients receiving tracheostomy.

\section{Conclusions}

To the best of our knowledge, we present the largest published study of COVID-19 tracheostomy patients. Our findings of better adjusted in-hospital mortality rates for COVID-19 critical care patients that had tracheostomies compared to patients without, provides evidence that the procedure is safe and advantageous for this cohort of patients. Data on tracheostomy use and outcomes for patients with a tracheostomy will assist hospitals prepare for further waves of COVID-19 patients in the future.

\section{REFERENCES}

1 Karagiannidis C., Mostert C., Hentschker C., et al. (2020) Case characteristics, resource use, and outcomes of 10021 patients with COVID-19 admitted to 920 German hospitals: an observational study.Lancet Respir. Med. 0 .

2 Docherty A.B., Harrison E.M., Green C.A., et al. (2020) Features of 20133 UK patients in hospital with covid-19 using the ISARIC WHO Clinical Characterisation Protocol: Prospective observational cohort study. BMJ 369 .

3 Givi B., Schiff B.A., Chinn S.B., et al. (2020) Safety Recommendations for Evaluation and Surgery of the Head and Neck during the COVID-19 Pandemic. JAMA Otolaryngol. - Head Neck Surg.146 , 579-584. 
4 Michetti C.P., Burlew C.C., Bulger E.M., et al. (2020) Performing tracheostomy during the Covid-19 pandemic: Guidance and recommendations from the Critical Care and Acute Care Surgery Committees of the American Association for the Surgery of Trauma. Trauma Surg. Acute Care Open $\mathbf{5}$.

5 McGrath B.A., Brenner M.J., Warrillow S.J., et al. (2020) Tracheostomy in the COVID-19 era: global and multidisciplinary guidance.Lancet Respir. Med. 8 , 717-725.

6 Schultz M.J., Teng M.S. \& Brenner M.J. (2020) Timing of tracheostomy for patients with Covid-19 in the ICU-setting precedent in unprecedented times. JAMA Otolaryngol. - Head Neck Surg. 146 , 887-888.

7 Lamb C.R., Desai N.R., Angel L., et al. (2020) Use of Tracheostomy During the COVID-19 Pandemic: American College of Chest Physicians/American Association for Bronchology and Interventional Pulmonology/Association of Interventional Pulmonology Program Directors Expert Panel Report. Chest $158,1499-1514$.

8 Takhar A., Tornari C., Amin N., et al. (2020) Safety and outcomes of percutaneous tracheostomy in coronavirus disease 2019 pneumonitis patients requiring prolonged mechanical ventilation.J. Laryngol. Otol. $134,961-970$.

9 Rovira A., Dawson D., Walker A., et al. (2020) Tracheostomy care and decannulation during the COVID-19 pandemic. A multidisciplinary clinical practice guideline. Eur. Arch. Oto-Rhino-Laryngology 278 .

10 Breik O., Nankivell P., Sharma N., et al. (2020) Safety and 30-day outcomes of tracheostomy for COVID19: a prospective observational cohort study. Br. J. Anaesth. 125, 872-879.

11 Hamilton N.J.I., Jacob T., Schilder A.G.M., et al. (2020) COVIDTrach; the outcomes of mechanically ventilated COVID-19 patients undergoing tracheostomy in the UK: Interim Report. Br. J. Surg.107 , e 583-e 584 .

12 NHS Digital (2013) Information Standard Board for Health and Social Care. Anonymisation Standard for Publishing Health and Social Care Data Specification (Process Standard) London, UK.

13 Navaratnam A. V, Gray W.K., Day J., et al. (2021) Patient factors and temporal trends associated with COVID-19 in-hospital mortality in England: an observational study using administrative data.

Lancet. Respir. Med.

14 Charlson M.E., Pompei P., Ales K.L., et al. (1987) A new method of classifying prognostic comorbidity in longitudinal studies: Development and validation. J. Chronic Dis. 40, 373-383.

15 Quan H., Li B., Couris C.M., et al. (2011) Updating and validating the charlson comorbidity index and score for risk adjustment in hospital discharge abstracts using data from 6 countries. Am. J. Epidemiol. $173,676-682$.

16 Staibano P., Levin M., McHugh T., et al. (2021) Association of Tracheostomy With Outcomes in Patients With COVID-19 and SARS-CoV-2 Transmission Among Health Care Professionals: A Systematic Review and Meta-analysis. JAMA Otolaryngol. Neck Surg. 147, 646-655.

17 Li M., Yiu Y., Merrill T., et al. (2018) Risk Factors for Posttracheostomy Tracheal Stenosis. Otolaryngol. - Head Neck Surg. (United States) 159, 698-704.

18 Cummings M.J., Baldwin M.R., Abrams D., et al. (2020) Epidemiology, clinical course, and outcomes of critically ill adults with COVID-19 in New York City: a prospective cohort study.Lancet 395 , 1763-1770.

19 Nouraei S.A.R., Hudovsky A., Frampton A.E., et al. (2015) A study of clinical coding accuracy in surgery: Implications for the use of administrative big data for outcomes management. Ann. Surg.261, 1096-1107.

Table 1: Profile of patients admitted to hospital, admitted to critical care and undergoing a tracheostomy 


\begin{tabular}{|c|c|c|}
\hline Variable & Hospital admissions $(n=132446)$ & Critical care admissions $(n=$ \\
\hline \multicolumn{3}{|l|}{ Age band (years) } \\
\hline $18-39$ & $11943(9.0 \%)$ & $1143(8.5 \%)$ \\
\hline $40-49$ & $10569(8.0 \%)$ & $1733(12.9 \%)$ \\
\hline $50-59$ & $17485(13.2 \%)$ & $3333(24.9 \%)$ \\
\hline $60-69$ & $20141(15.2 \%)$ & $3766(28.1 \%)$ \\
\hline 70-79 & $27951(21.1 \%)$ & $2684(20.0 \%)$ \\
\hline 80 & $44357(33.5 \%)$ & $742(5.5 \%)$ \\
\hline Sex & Sex & \\
\hline Female & $60139(45.5 \%)$ & $4272(32.2 \%)$ \\
\hline Male & $72081(54.5 \%)$ & $8980(67.8 \%)$ \\
\hline Missing & 226 & 149 \\
\hline Deprivation quintile & Deprivation quintile & \\
\hline 1 (most deprived) & $35456(27.4 \%)$ & $3675(28.0 \%)$ \\
\hline 2 & $28758(22.2 \%)$ & $3042(23.2 \%)$ \\
\hline 3 & $24179(18.7 \%)$ & $2485(19.0 \%)$ \\
\hline 4 & $21750(16.8 \%)$ & $2008(15.3 \%)$ \\
\hline 5 (least deprived) & $19314(14.9 \%)$ & $1894(14.5 \%)$ \\
\hline Missing & 2989 & 297 \\
\hline Ethnicity & Ethnicity & \\
\hline White & $94825(80.6 \%)$ & $7579(67.5 \%)$ \\
\hline South Asian or South Asian British & $8497(7.2 \%)$ & $1234(11.0 \%)$ \\
\hline Other Asian or other Asian British & $3155(2.7 \%)$ & $614(5.5 \%)$ \\
\hline Black or Black British & $6186(5.3 \%)$ & $959(8.5 \%)$ \\
\hline Mixed & $1031(0.9 \%)$ & $158(1.4 \%)$ \\
\hline Other ethnic groups & $3945(3.4 \%)$ & $682(6.1 \%)$ \\
\hline Missing & 14807 & 2175 \\
\hline Charlson Comorbidity Index items* & Charlson Comorbidity Index items* & \\
\hline Peripheral vascular disease & $6930(5.2 \%)$ & $560(4.2 \%)$ \\
\hline Congestive heart failure & $18970(14.3 \%)$ & $1405(10.5 \%)$ \\
\hline Acute myocardial infarction & $12152(9.2 \%)$ & $990(7.4 \%)$ \\
\hline Cerebrovascular disease & $12239(9.2 \%)$ & $736(5.5 \%)$ \\
\hline Dementia & $18931(14.3 \%)$ & $109(0.8 \%)$ \\
\hline Chronic pulmonary disease & $34889(26.3 \%)$ & $3132(23.4 \%)$ \\
\hline Connective tissue disease/rheumatic disease & $3933(3.0 \%)$ & $322(2.4 \%)$ \\
\hline Peptic ulcer & $896(0.7 \%)$ & $97(0.7 \%)$ \\
\hline Mild liver disease & $4446(3.4 \%)$ & $551(4.1 \%)$ \\
\hline Moderate or severe liver disease & $1467(1.1 \%)$ & $259(1.9 \%)$ \\
\hline Diabetes without chronic complications & $32078(24.2 \%)$ & $3762(28.1 \%)$ \\
\hline Diabetes with chronic complications & $3951(3.0 \%)$ & $375(2.8 \%)$ \\
\hline Paraplegia and hemiplegia & $3112(2.3 \%)$ & $189(1.4 \%)$ \\
\hline Renal disease & $23821(18.0 \%)$ & $1596(11.9 \%)$ \\
\hline Primary cancer & $7459(5.6 \%)$ & $517(3.9 \%)$ \\
\hline Metastatic carcinoma & $4076(3.1 \%)$ & $151(1.1 \%)$ \\
\hline HIV/AIDS & $197(0.1 \%)$ & $22(0.2 \%)$ \\
\hline Obesity & $12167(9.2 \%)$ & $2563(19.1 \%)$ \\
\hline
\end{tabular}

* For the Charlson Comorbidity Index items: Only those with the disease are listed. There were no missing data. Individual patients can appear in multiple disease categories.

Table 2: Multilevel logistic regression models of factors associated with critical care patients 
with a tracheostomy

\begin{tabular}{|c|c|}
\hline Variable & Odds ratios $(95 \%$ CIs $)$ \\
\hline \multicolumn{2}{|l|}{ Age band (years) } \\
\hline 18-39 (reference) & 1 (reference) \\
\hline $40-49$ & $2.118(1.605$ to 2.795$)$ \\
\hline $50-59$ & $2.375(1.837$ to 3.071$)$ \\
\hline $60-69$ & $2.193(1.693$ to 2.840$)$ \\
\hline 70-79 & $1.590(1.200$ to 2.107$) ? ¿ ?$ \\
\hline 80 & $0.249(0.134$ to 0.464$)$ \\
\hline \multicolumn{2}{|l|}{ Sex } \\
\hline Female & 1 (reference) \\
\hline Male & $1.222(1.073$ to 1.392$)$ \\
\hline Deprivation quintile & Deprivation quintile \\
\hline 5 (least deprived) & 1 (reference) \\
\hline 4 & $1.040(0.830$ to 1.304$)$ \\
\hline 3 & $1.021(0.822$ to 1.269$)$ \\
\hline 2 & $0.954(0.771$ to 1.180$)$ \\
\hline 1 (most deprived) & $0.857(0.691$ to 1.062$)$ \\
\hline \multicolumn{2}{|l|}{ Ethnicity } \\
\hline White & 1 (reference) \\
\hline South Asian & $1.328(1.071$ to 1.647$)$ \\
\hline Other Asian & $1.418(1.102$ to 1.824$)$ \\
\hline Black & $1.279(1.018$ to 1.607$)$ \\
\hline Mixed & $1.469(0.931$ to 2.317$)$ \\
\hline Other & $1.258(0.986$ to 1.605$)$ \\
\hline \multicolumn{2}{|l|}{ Discharge month } \\
\hline March & 1 (reference) \\
\hline April & $3.356(2.020$ to 5.574$)$ \\
\hline May & $19.067(11.502$ to 31.608$)$ \\
\hline June & $31.394(18.734$ to 52.609$)$ \\
\hline July & $23.842(13.927$ to 40.816$)$ \\
\hline August & $14.988(8.190$ to 27.428$)$ \\
\hline September & $5.200(2.764$ to 9.783$)$ \\
\hline October & $1.863(1.033$ to 3.362$)$ \\
\hline \multicolumn{2}{|l|}{ Charlson Comorbidity Index items* } \\
\hline Peripheral vascular disease & $0.723(0.524$ to 0.997$)$ \\
\hline Congestive heart failure & $0.498(0.393$ to 0.631$)$ \\
\hline Acute myocardial infarction & $0.581(0.444$ to 0.759$)$ \\
\hline Cerebrovascular disease & $1.946(1.539$ to 2.462$)$ \\
\hline Dementia & $0.657(0.294$ to 1.471$)$ \\
\hline Chronic pulmonary disease & $1.031(0.894$ to 1.189$)$ \\
\hline Connective tissue disease/rheumatic disease & $0.636(0.413$ to 0.978$)$ \\
\hline Peptic ulcer & $1.214(0.651$ to 2.262$)$ \\
\hline Mild liver disease & $0.861(0.648$ to 1.144$)$ \\
\hline Moderate or severe liver disease & $0.606(0.374$ to 0.980$)$ \\
\hline Diabetes without chronic complications & $0.876(0.762$ to 1.005$)$ \\
\hline Diabetes with chronic complications & $0.795(0.550$ to 1.150$)$ \\
\hline Paraplegia and hemiplegia & $0.973(0.778$ to 1.217$)$ \\
\hline Renal disease & $0.839(0.755$ to 0.933$)$ \\
\hline Primary cancer & $0.495(0.348$ to 0.705$)$ \\
\hline
\end{tabular}




\begin{tabular}{ll}
\hline Variable & Odds ratios $(\mathbf{9 5 \%}$ CIs $)$ \\
\hline Metastatic carcinoma & $\mathbf{0 . 1 4 1}(\mathbf{0 . 0 5 5}$ to $\mathbf{0 . 3 6 3 )}$ \\
Obesity & $0.923(0.788$ to 1.081$)$ \\
\hline
\end{tabular}

Models are based on data for 10,935 patients with no missing data.* For the Charlson Comorbidity Index items: Only those with the disease are listed. Individual patients can appear in multiple disease categories. HIV/AIDS was not included in the model due to the small number of patients with this condition receiving tracheostomy.

Table 3: Outcomes for those with and without a tracheostomy within the critical care population

\begin{tabular}{llll}
\hline & Tracheostomy & No tracheostomy & Adjusted association \\
\hline In-hospital mortality (\%) & $473(21.5 \%)$ & $4718(42.1 \%)$ & Odds ratio: 0.514 (95\% CI 0.443 \\
Median length of critical care stay (IQR) & $31(22$ to 43$)$ & $6(3$ to 12$)$ & Odds ratio for length of stay $>8$ \\
\hline
\end{tabular}

$\mathrm{CI}=$ confidence interval.

Table 4: Timing of tracheostomy for those who survived to discharge and those who died

\begin{tabular}{ll}
\hline & Survived to discharge (n= 1,421) \\
\hline & Tracheostomy [?] 14 days post admission to critic \\
Median days hospital stay (IQR) & $37(27$ to 52$)$ \\
Median days critical care stay (IQR) & $28(22$ to 38$)$ \\
Median days from tracheostomy to critical care discharge/death (IQR) & $15.5(10$ to 26$)$ \\
Median days from tracheostomy to discharge/death (IQR) & $27(18$ to 41$)$ \\
\hline
\end{tabular}

$\mathrm{IQR}=$ inter-quartile range

Figure 1: Adjusted percentage tracheostomy use in critical care patients and adjusted mortality rate in those with a tracheostomy by month of discharge

Figure 2: Percentage tracheostomy use in critical care patients over time by region

\section{Hosted file}

Figure 1.docx available at https://authorea.com/users/335032/articles/548454-utilisationof-tracheostomy-in-patients-with-covid-19-in-england-patient-characteristics-timing-andoutcomes

\section{Hosted file}

Figure 2.docx available at https://authorea.com/users/335032/articles/548454-utilisationof-tracheostomy-in-patients-with-covid-19-in-england-patient-characteristics-timing-andoutcomes 